\title{
A randomized trial of use of a modified reverse sural neurofasciocutaneous flap to extend the reconstruction range
}

\author{
Xin Huang, Jihua Xu, Hu Yang, Haifei Shi \\ Department of Orthopedics, The First Affiliated Hospital, Zhejiang University School of Medicine, Hangzhou, China \\ Contributions: (I) Conception and design: X Huang, J Xu; (II) Administrative support: H Shi; (III) Provision of study materials or patients: X Huang, J \\ Xu, H Shi; (IV) Collection and assembly of data: H Yang; (V) Data analysis and interpretation: X Huang; (VI) Manuscript writing: All authors; (VII) \\ Final approval of manuscript: All authors. \\ Correspondence to: Haifei Shi. Department of Orthopedics, The First Affiliated Hospital, Zhejiang University School of Medicine, Hangzhou 310003 , \\ China. Email: shihaifei@zju.edu.cn.
}

Background: When a reverse sural neurofasciocutaneous flap is used to reconstruct a forefoot defect, usually, the transverse length of the flap is increased or the pivot point is lowered to ensure the reconstruction range. Therefore, proximal partial necrosis leading to surgery failure is sometimes caused by insufficient arterial supply if the flap is too long or the pivot point is too low and has no a reliable perforator in the pedicle. Herein, we describe a new method for extending the reconstruction range of the reverse sural neurofasciocutaneous flap that can provide a higher survival rate.

Methods: Between July 2010 and July 2014, 24 patients with forefoot defect were randomly assigned into two groups. In the research group, 12 modified reverse sural neurofasciocutaneous flaps with extended reconstruction range were used to reconstruct forefoot defect. We dissected the septum along the last perforator to locate the main trunk of the peroneal artery, disconnected the proximal end of the peroneal artery at the root of the perforator, and separated the peroneal artery more distally to obtain a lower rotation point to extend the reconstruction range. The last perforator and a section of the peroneal artery were contained in the pedicle. In the control group, 12 traditional reverse sural neurofasciocutaneous flap procedures were performed.

Results: In the research group, 11 flaps survived with good quality and esthetic contours. Partial distal necrosis occurred in 1 flap. In the control group, 7 flaps survived. Partial necrosis occurred in the other 5 flaps. Compared with control group, the survival rate in the research group was significantly higher while the healing time was significantly shorter. All patients were satisfied with the therapeutic outcome in the research group.

Conclusions: Our modified method proved efficacious in extending the reconstruction range of the reverse sural neurofasciocutaneous flap. Meanwhile, partial necrosis was avoided because the length of the flap was shortened, and a reliable perforator was preserved in the artery pedicle.

Trial Registration: Chictr.org Identifier: ChiCTR2100046323.

Keywords: Perforator flap; reverse flap; sural flap; forefoot reconstruction

Submitted Dec 28, 2020. Accepted for publication Jun 15, 2021.

doi: 10.21037/atm-21-1442

View this article at: https://dx.doi.org/10.21037/atm-21-1442 


\section{Introduction}

The reverse sural neurofasciocutaneous flap is widely used to reconstruct soft-tissue defects of the foreleg (1-5), but reconstruction of the forefoot remains a challenge for plastic surgeons. To extend the reconstruction range of the flap, plastic surgeons have two options: increase the length of the flap (6-8) or lower the pivot point (9-11).

Traditionally, a subcutaneous fascial pedicle should be elevated with a sufficient width to ensure an adequate blood supply to the flap. The longer the flap is, the wider the pedicle must be $(1,12)$. Even so, partial necrosis at the proximal end of the flap sometimes occurs because of an insufficient link-pattern blood supply $(7,13)$. As an alternative strategy, plastic surgeons and researchers have conducted anatomical research to identify a lower peroneal perforator in an attempt to lower the rotation point of the flap and decrease the flap length. Al-Qattan (6) described a low pivot point sural neurofasciocutaneous flap based on perforators from the lateral calcaneal artery and posterior lateral malleolus artery. However, in our clinical experience, these perforators are not a reliable arterial source for such a large flap because of their small diameter and instability.

We generally use the proximal part of the reverse sural flap to reconstruct defects in the forefoot. However, if the flap is too long, this part sometimes has an insufficient arterial supply, which may cause partial necrosis. Postoperative necrosis in the proximal part leads to failure of the operation. For one, the distance from the perforator to the flap tip should be shortened to ensure the blood supply of the proximal part; for another, the pivot point should be lowered to ensure the reconstruction range can cover the wound in forefoot. These requirements led us to devise a plan that involves a modified reverse sural flap with the last perforator and a section of the peroneal artery in the pedicle to meet the repair requirement. In this approach, the length of the flap is shortened to obtain a reliable blood supply, and the rotation point is lowered to obtain a sufficient range. The article represents the first description of our modified method and a report of its performance for reconstruction of soft-tissue defects of the forefoot.

We present the following article in accordance with the CONSORT reporting checklist (available at https://dx.doi. org/10.21037/atm-21-1442).

\section{Methods}

\section{Trial design}

This study was a double blind randomized controlled trial.
Since the wounds of the lower limbs can not be repaired by direct suture and skin grafting, we choose reverse sural flap to repair the wounds of the lower limbs. Patients were allocated to one of two groups. The first group underwent our modified reverse sural neurofasciocutaneous flap fixation, the second group underwent traditional reverse sural flap fixation. According to the protocol of the study, five phases were followed:

* Debridement and clinical assessment;

* Randomly assigned into two groups;

* Treated with surgery;

* Intraoperative and postoperative observation and measurement;

- Follow-up 12 to 24 months after surgery.

\section{Sample size}

The study was powered to detect clinically significant difference in the survival rate of the modified and traditional reverse sural flap using a $=0.05$, a power $=95 \%$. When we estimate that the wounds of the lower limbs which could not be repaired by direct suture and skin grafting, we repaired the wounds by flap. Based on previous studies, there was nearly zero mortality and zero drop-out. As a minimum, 12 patients were enrolled in each group according to $1: 1$ randomized allocation.

\section{Randomisation}

Patients were assigned to one of the treatment groups with the use of a computer-generated randomization table. Each patient participated in modified or traditional reverse sural flap surgery. Allocation concealment was obtained using sealed coded opaque envelopes containing the operation to the specific subject. The envelope was opened during the surgery after the wounds of the lower limbs were debrided.

\section{Patients}

This report describes the clinical course of 24 patients with a soft-tissue defect of the lower extremity [18 males, 6 females; 21-65 (mean, 33.8) years old]. The patients were randomly assigned into two groups. Twelve patients were treated with our modified reverse sural neurofasciocutaneous flap technique to repair the foot wound and to recover function of the extremity. The other 12 patients in the control group were treated with the traditional reverse sural flap technique. All patients 
Table 1 The data and follow-up of the modified reverse sural neurofasciocutaneous flap

\begin{tabular}{lccc}
\hline $\begin{array}{l}\text { Last perforator } \\
\text { above the lateral } \\
\text { malleolus tip }(\mathrm{cm})\end{array}$ & $\begin{array}{c}\text { Pivot point above } \\
\text { the lateral malleolus } \\
\text { tip }(\mathrm{cm})\end{array}$ & $\begin{array}{c}\text { Follow-up } \\
\text { (months) }\end{array}$ & Result \\
\hline 7 & 3 & 18 & Healed \\
6.3 & 2 & 12 & Healed \\
8 & 4 & 12 & Healed \\
7 & 3 & 18 & Healed \\
7.5 & 4 & 12 & Healed \\
8 & 4 & 12 & Healed \\
6 & 2 & 24 & Healed \\
7.4 & 3 & 18 & Healed \\
7 & 4 & 12 & Healed \\
7.8 & 4 & 12 & Healed \\
\hline
\end{tabular}

underwent surgery between July 2010 and July 2014. All wounds (8 on the dorsum and 16 on the plantar surface) required surgical intervention because of infection or osseous and tendon exposure. In 9 cases, the distal edge of the wound reached the proximal metatarsal bone, whereas in the other 13 cases, it reached the metatarsophalangeal joint. The size of the defects arranged from $5 \mathrm{~cm} \times 4 \mathrm{~cm}$ to $27 \mathrm{~cm} \times 11 \mathrm{~cm}$. Details of the patients and wounds are described in Table 1.

All procedures performed in this study involving human participants were in accordance with the Declaration of Helsinki (as revised in 2013). The study was approved by the Ethics Committee of The First Affiliated Hospital, Zhejiang University School of Medicine (No. 2021188 ) and written informed consent was obtained from all patients.

\section{Surgical technique}

All operations were performed under continuous epidural anesthesia. The patient was placed in the prone position, and a tourniquet was used. The primary surgical plan was to reconstruct the defect with a reverse sural neurofasciocutaneous flap. An imaginary line was drawn from the midline of the popliteal fossa to the midpoint between the lateral malleolus and the Achilles tendon. The last perforator was marked on the line as the primary pivot point, which was usually $5-7 \mathrm{~cm}$ above the tip of the medial

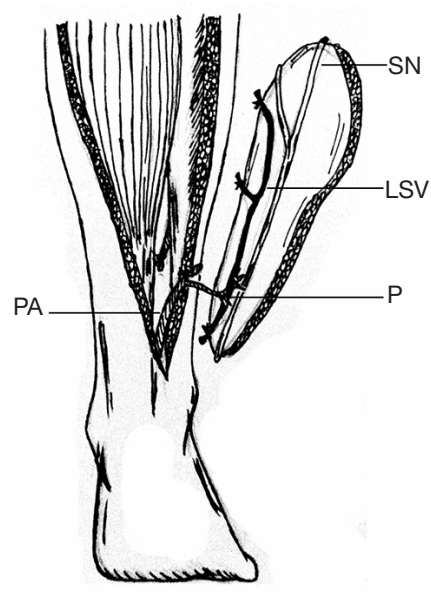

Figure 1 Design of the modified reverse sural neurofasciocutaneous flap. PA, peroneal artery; LP, last perforator of peroneal artery; LSV, lesser saphenous vein; SN, sural nerve.

malleolus. The flap was designed according to the location and size of the defect. The distance from the pivot point to the proximal point of the flap was the same as the distance from the pivot point to the distal margin of the wound. With a proximal incision, the flap was dissected to the deep fascia. In regards to the sural nerve, the proximal part of the flap was elevated rapidly from the subfascial plane to ensure that the neurovascular structure was preserved in the flap.

Once the dissection reached the distal part of the flap, we paid special attention to identify the distal perforators. One or two distal perforators were separated, and the last perforator was preserved. The soft tissue of the pedicle was thinned, with some adipofascial tissue being preserved around the perforator to create a propeller form. The viability of the flap was assessed after the tourniquet was released.

In the 12 cases described in this report, we found that the proximal arterial supply was unsatisfactory. We therefore removed the ischemic part to ensure flap survival. However, we encountered problem in which the proximal edge of the flap could not reach the distal edge of the wound if the flap length was decreased. Therefore, we had to lower the pivot point. Further intraseptal dissection was performed to expose the main trunk of the peroneal artery. We disconnected the proximal end of the peroneal artery at the root of the perforator and dissected the peroneal artery distally to create a lower pivot point. Dissection was performed until the range of the flap extended to the distal margin of the defect (Figure 1). If the distance from the root 


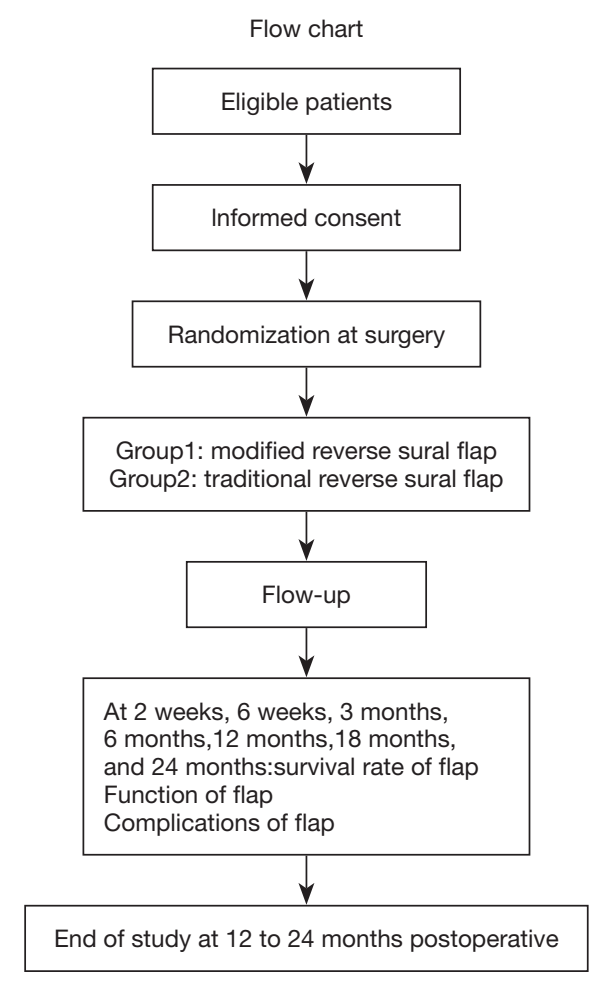

Figure 2 Follow up schedule.

of the last perforator to the actual pivot point was $2 \mathrm{~cm}$, the flap was extended $4 \mathrm{~cm}$ further. After a $180^{\circ}$ rotation, the flap was sutured into the defect. The donor site was closed by primary suturing or full-thickness skin grafting.

\section{Blinding}

Both patients and surgeons would be blinded regarding which method of flap was used. The surgeon performing the procedure was obviously not blinded; surgeons performing the assessments during follow up would be blinded to the surgery. A randomization number was issued and was noted in patients' files. The operating report would not specify which operation had been used. The following statement was noted at the end of the operating report: 'The trial participant was randomized in 1 of the 2 surgeries of the Trial. For blinding purposes, this data had been stored in a secured database. Postoperatively, patients were not informed of the surgery of flap that was used. Patients and surgeons would be questioned during every postoperative follow up appointment on the presumed surgery. In this fashion, one would be able to assess whether double blind randomization was realistic upon evaluation. As patients and surgeons were blinded to the surgery; the survival rate of flap in trial patients would not be assessed by their own surgeons during follow up.

\section{Follow up}

The follow-up period would be 12 to 24 months after surgery. Patients would visit the outpatient department for evaluation at 2 weeks, 6 weeks, 3 months, 6 months, 12 months, 18 months and 24 months postoperatively. A figure of the follow-up schedule is visible in Figure 2. Subjects could leave the study at any time for any reason if they wish to do so without any consequences. The investigator could decide to withdraw a subject from the study for urgent medical reasons. Table 1 represents the follow-up of the modified reverse sural neurofasciocutaneous flap.

\section{Statistical analysis}

Data analysis was performed according to the intention-totreat principle for all randomized patients from July 2010 to July 2014. The distance of the last perforator and the pivot point above the lateral malleolus tip were measured. We compared the survival rate of flap within each trial. Statistical analysis was undertaken using SPSS, version 23.0 (IBM Corp.). Results were used paired $t$-tests, to establish if there was statistically significant between modified and traditional reverse sural neurofasciocutaneous flaps.

\section{Results}

Postoperative follow-up over a period of 12 to 24 months revealed that 11 flaps survived with good quality and esthetic contours in the research group, with partial distal necrosis occurring in 1 flap. The wound healed with the treatment of surgical debridement and skin graft. No sign of ischemia in the distal extremities due to sacrificing the peroneal artery was observed postoperatively. There were no complaints related to sacrificing the sural nerve. All patients were satisfied with their therapeutic outcome. In the control group, 7 flaps survived, with partial necrosis occurring in the other 5 flaps. Compared with control group, the survival rate of the research group was significantly higher $(\mathrm{P}<0.001)$, while the healing time was significantly shorter (Table 2 ). 


\section{Case presentation}

\section{Case 1}

A 41-year-old man sustained an injury to his left foot in a motorcycle accident 4 hours before presentation at our department, resulting in skin avulsion on the dorsum pedis. After thorough debridement, the skin defect measured $10 \mathrm{~cm} \times 7 \mathrm{~cm}$. A reverse sural neurofasciocutaneous flap was designed to repair the defect. During the operation, we found the proximal arterial supply to be unsatisfactory.

Table 224 patients were randomly assigned into two groups

\begin{tabular}{lcc}
\hline Variable & $\begin{array}{c}\text { Modified reverse sural } \\
\text { neurofasciocutaneous } \\
\text { flap }\end{array}$ & $\begin{array}{c}\text { Traditional } \\
\text { reverse sural } \\
\text { flap }\end{array}$ \\
\hline $\begin{array}{l}\text { Underwent surgery } \\
\text { Postoperative follow-up }\end{array}$ & 12 & 12 \\
$\begin{array}{l}\text { Good quality and } \\
\text { esthetic contours }\end{array}$ & 11 & 7 \\
$\begin{array}{l}\text { Partial distal necrosis } \\
\text { occurring }\end{array}$ & 1 & 5 \\
\hline
\end{tabular}

Intraseptal dissection was performed to expose the main trunk of the peroneal artery. We removed the ischemic part to ensure survival of the flap, and noted that the flap could not reach the distal edge of the defect. We subsequently disconnected the proximal end of the peroneal artery at the root of the perforator and dissected the peroneal artery distally to make a lower pivot point. Dissection was performed until the range of the flap extended to the distal margin of the defect. The pivot point was lowered by $4 \mathrm{~cm}$. The flap reached $8 \mathrm{~cm}$ further. With a $180^{\circ}$ rotation, the flap was sutured into the defect. The donor site was closed by full-thickness skin grafting. At 18-month followup, the flap was noted to have survived, and it exhibited a satisfactory contour (Figure 3).

\section{Case 8}

A 28-year-old man sustained a crush injury over his left lateral heel in a car accident. After thorough debridement, there was an $11 \mathrm{~cm} \times 5 \mathrm{~cm}$ skin defect and accompanying bone exposure. Two weeks after the injury, a reverse sural neurofasciocutaneous flap was designed to repair the defect. During surgery, we noted that the proximal arterial supply
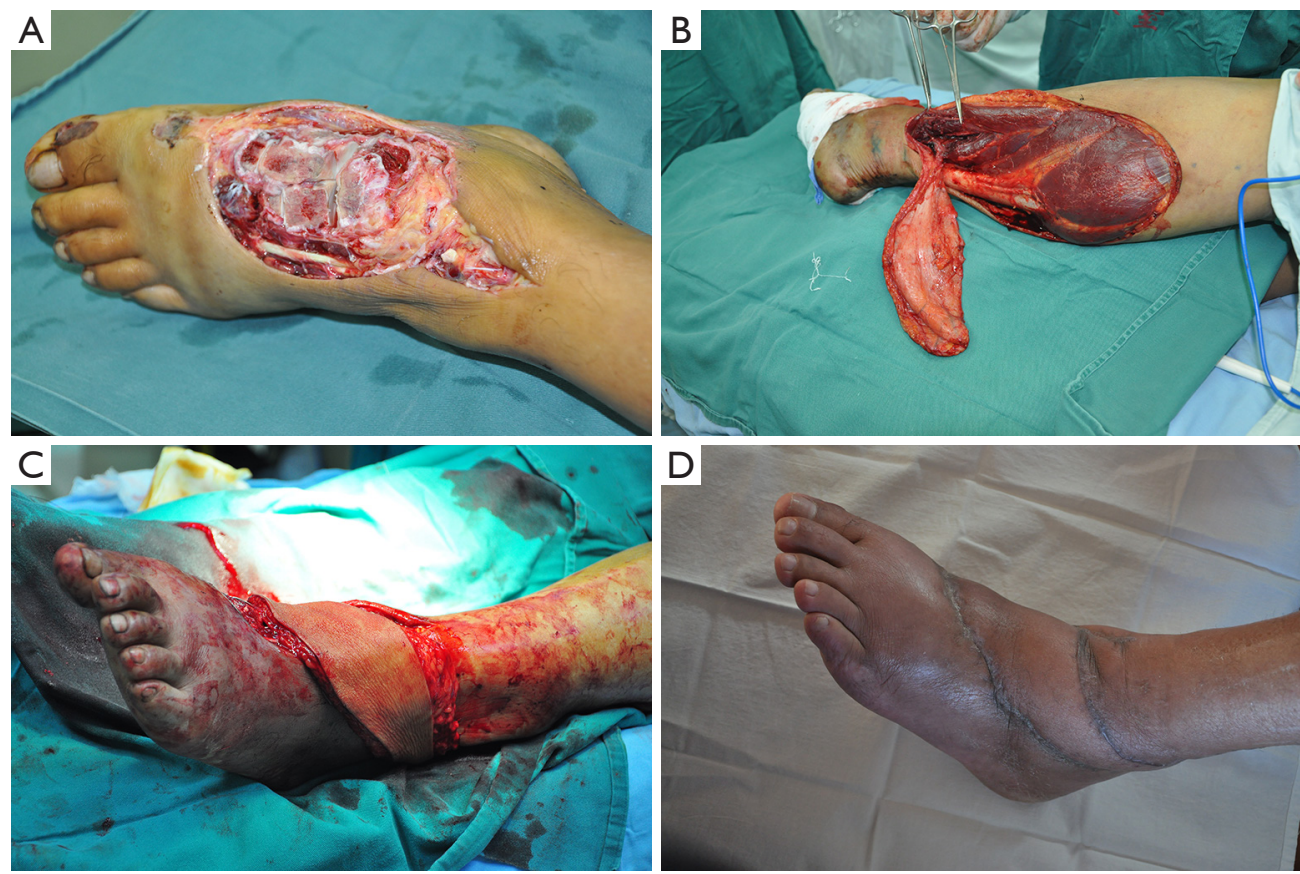

Figure 3 Presentation of Case 1. (A) A skin defect measuring $10 \mathrm{~cm} \times 7 \mathrm{~cm}$ over left dorsum pedis. (B) A reverse sural neurofasciocutaneous flap was elevated. The proximal end of the peroneal artery was disconnected to lower the pivot point. The pivot point was located 3 cm above the lateral malleolus tip. (C) The flap was sutured into the defect with a $180^{\circ}$ rotation. The donor site was closed by full-thickness skin grafting. (D) The 18-month follow-up showed that the flap survived with a satisfactory contour. 

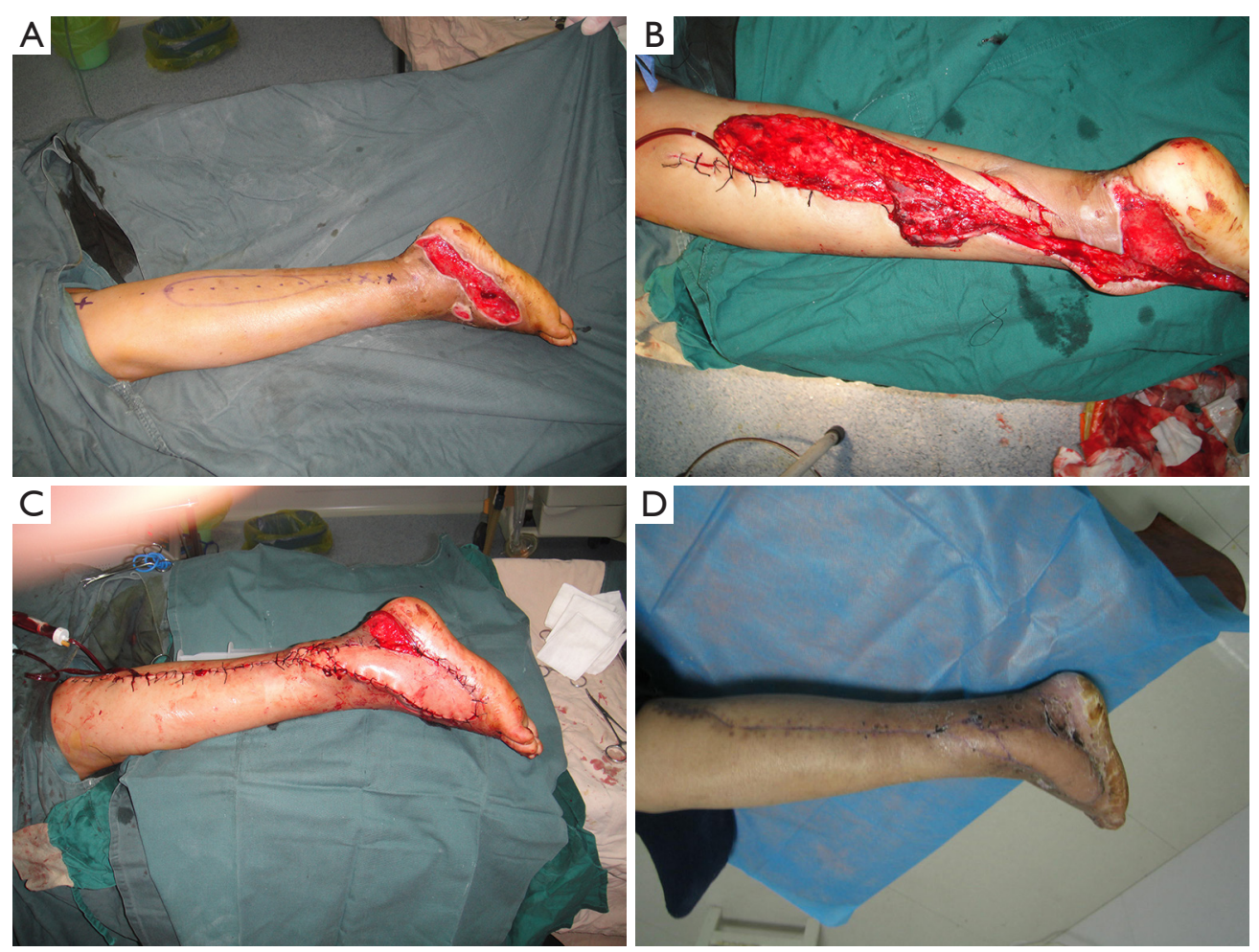

Figure 4 Presentation of Case 8. (A) A skin defect measuring $11 \mathrm{~cm} \times 5 \mathrm{~cm}$ over left lateral heel, along with the flap design. (B) Exposure of the peroneal artery and its last perforator. (C) The flap was sutured into the defect with a $180^{\circ}$ rotation. The pivot point was located $2 \mathrm{~cm}$ above the lateral malleolus tip. The donor site was primarily closed without a skin graft. (D) The 24-month follow-up showed a satisfactory result with normal walking.

was unsatisfactory. Intraseptal dissection was performed to expose the main trunk of the peroneal artery. We removed the ischemic part to ensure survival of the flap and lowered the pivot point by $4 \mathrm{~cm}$. The flap was moved $8 \mathrm{~cm}$ further to reach the distal edge of the defect. The donor site was closed without a skin graft. At 24-month follow-up, the results were satisfactory, and the patient was able to walk in a normal manner (Figure 4).

\section{Discussion}

Reconstruction of the forefoot with a reverse sural neurofasciocutaneous flap remains a challenge for plastic surgeons. For defects located at the distal part of the limb, the distance from the pivot point to the distal margin of the wound is long. To obtain a sufficient range for the flap, plastic surgeons have the option of increasing the length of the flap or trying to lower the pivot point.

There are problems with both options. For instance, Wei et al. (7) equally divided the posterior aspect of the lower leg into nine zones (ranging from number 1 distally to number 9 proximally), and based on the top edge of the flap, reverse sural neurofasciocutaneous flaps were classified into four groups. The authors concluded that the partial necrosis ratio tended to increase as the location of the top edge became more proximal. Zhang et al. (9) described a low pivot point sural neurofasciocutaneous flap based on perforators from the lateral calcaneal artery and posterior lateral malleolar artery. However, according to our clinical experience, these perforators are not a reliable source of arterial blood flow for such a large flap because of their small diameter and instability. Furthermore, the lower perforator is sometimes too close to the wound, which further reduces its reliability as an arterial blood supply.

In our study, to decrease partial necrosis ratio, we assessed the viability of the reverse sural neurofasciocutaneous flap intraoperatively after the tourniquet was released. We removed the ischemic tissue in the proximal part of the flap to ensure that the arterial supply of the rest of the tissue 
A

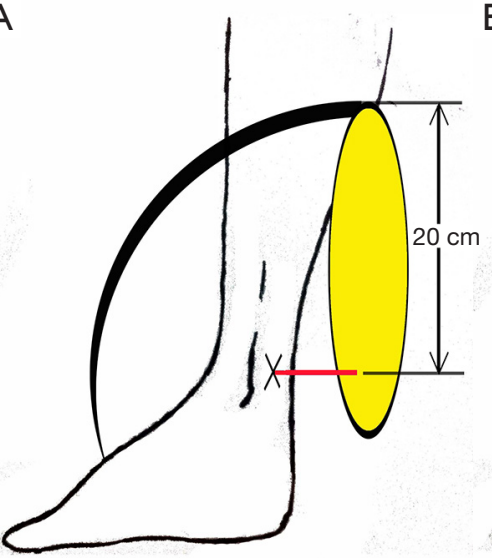

B

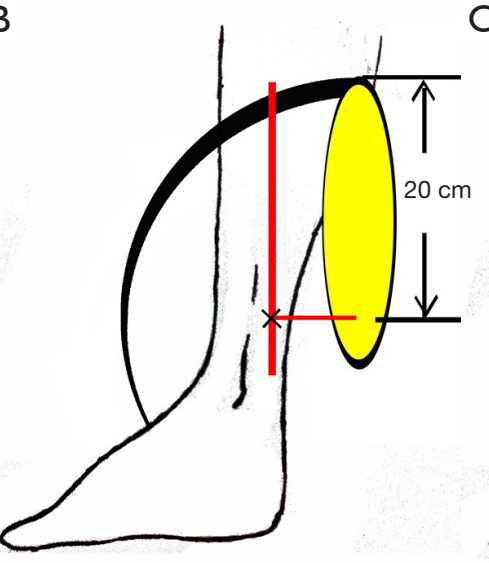

C

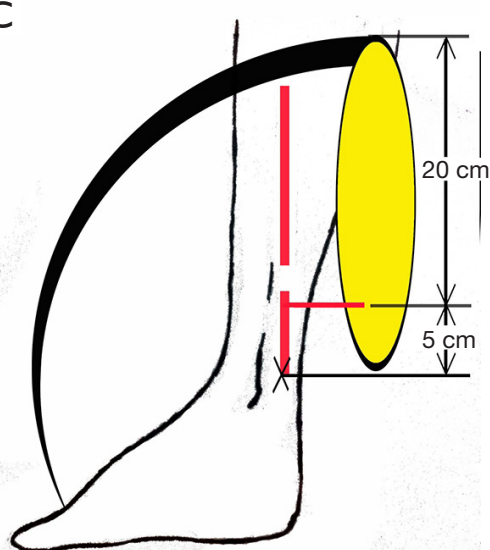

Figure 5 The difference of the reconstruction range of the flaps. (A) The reconstruction range of the reverse sural flap based on the retromalleolar perforator. (B) The reconstruction range of the reverse sural flap based on last perforator of the peroneal artery. (C) The reconstruction range of our flap.

was reliable. To make certain that the flap reached the distal edge of the defect, we used the main trunk of the peroneal artery to lower the pivot point. If the distance from the root of last perforator to the actual pivot point was $2 \mathrm{~cm}$, the flap would reach $4 \mathrm{~cm}$ further (Figure 5).

At our institution, we generally prefer to design a perforator pedicle propeller flap, which is defined as a flap nourished by a perforator vessel. The perforator is skeletonized and freed from fascial adhesions. The flap can then be rotated safely, without strangulation of the pedicle $(14,15)$.

We are first to report a modified method in which the peroneal artery is disconnected from the level of its last perforator. Although the artery supplies the ankle and heel below this level, anatomic studies have indicated that there is a rich network of vessels over the Achilles tendon and heel, formed by branches of the posterior tibial and peroneal arteries $(16,17)$. Therefore, sacrifice of the peroneal artery from the lateral malleolus level will not increase the morbidity of the ankle and heel. In our cases, we observed no postoperative necrosis due to sacrifice of the peroneal artery.

\section{Conclusions}

Our modified method proved efficacious for extending the reconstruction range of the reverse sural neurofasciocutaneous flap. Meanwhile, partial necrosis was avoided by shortening the length of the flap, and a reliable perforator was preserved in the artery pedicle. Compared with the traditional method, our modified method yielded a markedly higher survival rate. We believe this novel approach can offer plastic surgeons a means to performing a modified reverse sural neurofasciocutaneous flap technique that provides an extended reconstruction range.

\section{Acknowledgments}

Funding: None.

\section{Footnote}

Reporting Checklist: The authors have completed the CONSORT reporting checklist. Available at https://dx.doi. org/10.21037/atm-21-1442

Trial Protocol: Available at https://dx.doi.org/10.21037/atm21-1442

Data Sharing Statement: Available at https://dx.doi. org/10.21037/atm-21-1442

Conflicts of Interest: All authors have completed the ICMJE uniform disclosure form (available at https://dx.doi. org/10.21037/atm-21-1442). The authors have no conflicts of interest to declare.

Ethical Statement: The authors are accountable for all aspects of the work in ensuring that questions related to the accuracy or integrity of any part of the work are 
appropriately investigated and resolved. The study was approved by the Ethics Committee of The First Affiliated Hospital, Zhejiang University School of Medicine (No. 2021-188) and written informed consent was obtained from all patients. All procedures performed in this study involving human participants were in accordance with the Declaration of Helsinki (as revised in 2013).

Open Access Statement: This is an Open Access article distributed in accordance with the Creative Commons Attribution-NonCommercial-NoDerivs 4.0 International License (CC BY-NC-ND 4.0), which permits the noncommercial replication and distribution of the article with the strict proviso that no changes or edits are made and the original work is properly cited (including links to both the formal publication through the relevant DOI and the license). See: https://creativecommons.org/licenses/by-nc-nd/4.0/.

\section{References}

1. Masquelet AC, Romana MC, Wolf G. Skin island flaps supplied by the vascular axis of the sensitive superficial nerves: Anatomic study and clinical experience in the leg. Plast Reconstr Surg 1992;89:1115-21.

2. Hasegawa M, Torii S, Katoh H, et al. The distally based superficial sural artery flap. Plast Reconstr Surg 1994;93:1012-20.

3. Le Fourn B, Caye N, Pannier M. Distally based sural fasciomuscular flap: anatomic study and application for filling leg or foot defects. Plast Reconstr Surg 2001;107:67-72.

4. Touam C, Rostoucher P, Bhatia A, et al. Comparative study of two series of distally based fasciocutaneous flap for coverage of the lower one-fourth of the leg, the ankle, and the foot. Plast Reconstr Surg 2001;107:383-92.

5. Chen SL, Chen TM, Chou TD, et al. Distally based sural fasciomusculocutaneous flap for chronic calcaneal osteomyelitis in diabetic patient. Ann Plast Surg 2005;54:44-8.

6. Al-Qattan MM. A modified technique for harvesting the reverse sural artery flap from the upper part of the leg: inclusion of a gastrocnemius muscle "cuff" around the sural pedicle. Ann Plast Surg 2001;47:269-74.

Cite this article as: Huang $\mathrm{X}, \mathrm{Xu} \mathrm{J}$, Yang $\mathrm{H}$, Shi $\mathrm{H}$. A randomized trial of use of a modified reverse sural neurofasciocutaneous flap to extend the reconstruction range. Ann Transl Med 2021;9(12):1000. doi: 10.21037/atm-21-1442
7. Wei JW, Dong ZG, Ni JD, et al. Influence of flap factors on partial necrosis of reverse sural artery flap: A study of 179 consecutive flaps. J Trauma Acute Care Surg 2012;72:744-50.

8. Liu L, Zou L, Li Z, et al. The Extended Distally Based Sural Neurocutaneous Flap for Foot and Ankle Reconstruction: A Retrospective Review of 10 Years of Experience. Ann Plast Surg 2014;72:689-94.

9. Zhang FH, Chang SM, Lin SQ, et al. Modified distally based sural neuroveno-fasciocutaneous flap: anatomical study and clinical applications. Microsurgery 2005;25:543-50.

10. Zhang F, Zheng H, Xie Q, et al. Application anatomy of perforating branches of artery and its distally based flap of sural nerve-lesser saphenous vein. Zhongguo Xiu Fu Chong Jian Wai Ke Za Zhi 2005;19:501-4.

11. Zhang F, Lin S, Song Y, et al. Distally based sural neurolesser saphenous veno-fasciocutaneous compound flap with a low rotation point: microdissection and clinical application. Ann Plast Surg 2009;62:395-404.

12. Tu YK, Ueng SW, Yeh WL, et al. Reconstruction of ankle and heel defects by a modified wide-base reverse sural flap. J Trauma 1999;47:82-8.

13. Chang SM, Hou CL. Chain-linked directional vascular plexuses of the integument and link-pattern vascularized flaps in distal extremities. Plast Reconstr Surg 1998;101:2013-5.

14. Pignatti M, Ogawa R, Hallock GG, et al. The "Tokyo" consensus on propeller flaps. Plast Reconstr Surg 2011;127:716-22.

15. Chang SM, Wang X, Huang YG, et al. Distally based perforator propeller sural flap for foot and ankle reconstruction. Ann Plast Surg 2014;72:340-5.

16. Taylor GI, Pan WR. Angiosomes of the leg: anatomic study and clinical application. Plast Reconstr Surg 1998;102:599-616.

17. Attinger CE, Evans KK, Bulan E, et al. Angiosomes of the foot and ankle and clinical implications for limb salvage: reconstruction, incisions, and revascularization. Plast Reconstr Surg 2006;117:261S-293S.

(English Language Editor: J. Gray) 